\title{
Acute Kidney Injury Incidence, Stage, and Recovery in Patients with COVID-19
}

\author{
Lucie Bandelac', Kaanan D Shah', Pravish Purmessur², Haider Ghazanfar², Rabih Nasr ${ }^{2}$ \\ 'St. George's University School of Medicine, St. George, Grenada; ${ }^{2}$ Department of Internal Medicine, BronxCare Health System, Bronx, NY, USA \\ Correspondence: Lucie Bandelac, Department of Internal Medicine, 1650 Grand Concourse, Bronx, NY, 10457, USA, Email luciebandelac@gmail.com
}

\begin{abstract}
Purpose: To determine the incidence, mortality, stage, and recovery of acute kidney injury (AKI) in COVID-19 patients and further analyze the effect of patient demographics and comorbidities on AKI incidence.

Study Design: Our study looked at 1545 charts of patients over 18 years old who presented to BronxCare Hospital in NY with a positive SARS-CoV-2 PCR test. Using the KDIGO criteria, any patient presenting with a creatinine of 1.5 times the baseline or that had an increase in creatinine of $0.3 \mathrm{mg} / \mathrm{dL}$ in 48 hours was diagnosed with AKI. Pregnant patients, patients with end-stage renal disease (ESRD), and patients with a history of renal transplant were excluded.

Results: The incidence of AKI in COVID-19 patients was 39\% (608), and the mortality rate was $58.2 \%$ (354). Of the 254 survivors, $74.8 \%$ recovered. Moreover, $42.6 \%$ (259) of patients with AKI were admitted to the ICU. Twenty-six of our patients received hemodialysis during admission. There was a statistically significant association between AKI and age, race, hypertension (HTN), diabetes mellitus (DM), hepatitis C (HCV), congestive heart failure (CHF), CKD, patient outcome, and days spent in the hospital. Of the 608 patients with AKI, 294 (48.4\%), 185 (30.4\%) and 129 (21.2\%) had AKI stage 1, 2 and 3, respectively.

Conclusion: Early resource planning is necessary when admitting COVID-19 patients. Nephrology should be consulted early, and measures should be in place to optimize outpatient follow-up in the nephrology clinic. Lastly, the use of nephrotoxic agents should be carefully considered and, if possible, avoided from the time of admission in patients with COVID-19.
\end{abstract}

Keywords: acute kidney injury, AKI, COVID-19, hemodialysis, chronic kidney disease, CKD

\section{Introduction}

According to the CDC, COVID-19 cases have totaled over 70 million in the United States as of January $2022 .{ }^{1}$ Many studies have described pulmonary and extrapulmonary presentations of COVID-19 including but not limited to vasculitis in several organ systems. Although a renal phenotype has not yet been clearly defined, hematuria, proteinuria, and elevated creatinine levels have been reported in several studies. ${ }^{2,3}$ Several articles have been published worldwide and the incidence of AKI varies greatly. A meta-analysis on 20 articles published in June 2020 shows the incidence of AKI was approximately 9\% in COVID-19 patients. ${ }^{4}$ However, in an article published in January 2021, analyzing over 3000 patients across New York City, AKI was found to be present in $46 \%$ of the patients. ${ }^{5}$ In our study, we look at AKI throughout the first year of the pandemic on our local and underserved patient population in the Bronx, New York.

AKI is defined by a change in either serum creatinine, by $0.3 \mathrm{mg} / \mathrm{dL}$ or more within 48 hours, an increase in serum creatinine 1.5 times more than the baseline within the last 7 days, or urine output less than $0.5 \mathrm{~mL} / \mathrm{kg} / \mathrm{hr}$ for 6 hours. ${ }^{6}$ Clinically, we often look at urine output for diagnosis, as this does not require any prior patient data.

AKI can be isolated or superimposed on chronic renal disease. Risk factors for AKI include age above 65 and the presence of specific comorbidities such as hypertension, heart disease, liver disease, chronic kidney disease (CKD), peripheral artery disease, and diabetes. ${ }^{7}$ The causes of AKI can be distributed into pre-renal, renal, and post-renal causes. Different serum blood urea nitrogen to serum creatinine ratios can help localize the patient's AKI etiology.

To assess the severity of AKI, one must determine the ratio of creatinine to the baseline creatinine or the stage of AKI. If the creatinine at the time of AKI is 1.5 to 1.9 times the baseline, it is AKI Stage 1. If the creatinine is 2-2.9 times 
the baseline, it is AKI stage 2, and if the creatinine is above 2.9 times the baseline, it is AKI Stage 3. The stage of AKI is essential as it affects the treatment plan and prognosis of a patient. High-risk patients and stage 1 patients are treated preventatively by ensuring proper volume status, avoiding hyperglycemia and nephrotoxic agents, and monitoring creatinine and urine output. Stage 2 and 3 patients, however, require more aggressive measures. ICU admission and renal replacement therapy may be necessary. ${ }^{8}$ Although the underlying pathogenesis of AKI in patients with COVID-19 remains a subject of debate, associated comorbidities, medications, and viral insult to kidney cells are all possible causes.

\section{Method}

In our study, we retrospectively analyzed 1546 patient charts above 18 who were admitted to BronxCare hospital and tested positive for COVID-19 through a SARS-CoV-2 PCR test from March 2020 through December 2020. Pregnant patients, patients with end-stage renal disease on dialysis, and patients with a history of renal transplant were excluded. We then applied the KDIGO AKI criteria described in the introduction to the remaining patients to determine which patients had AKI and what stage of AKI they had. The baseline creatinine used was the most recent creatinine level recorded between 7 and 365 days prior to admission. If no such number was present in the patient's medical records, we calculated a standardized baseline using a GFR of $75 \mathrm{~mL} / \mathrm{min} / 1.37 \mathrm{~m}^{\wedge} 2$ through the MDRD equation (see below).

MDRD Equation: ${ }^{9}$

$$
\operatorname{GFR}\left(\frac{\frac{m l}{\min }}{1.37 m^{2}}\right)=186 \times \text { S.Cr } r^{-1.154} \times a g e^{-0.203} \times \text { GenderCoefficient } \times \text { RaceCoefficient }
$$

The gender coefficient is 1 for males, 0.742 for females.

The race coefficient was 1.21 for African-Americans and 1 for any other race.

Reversed MDRD Equation

$$
S . C r=\left[\frac{G F R\left(\mathrm{ml} / \mathrm{min} / 1.37 \mathrm{~m}^{2}\right)}{186 \times \text { age }{ }^{-0.203} \times \text { GenderCoefficient } \times \text { RaceCoefficient }}\right]^{(-1 / 1.154)}
$$

We further stratified our data to analyze the incidence, stage, and outcome of these AKI patients based on their demographics, comorbidities, and whether or not they were admitted into the ICU.

We calculated frequency and percentages for all qualitative variables. We used the Mann-Whitney $U$-test for continuous variables and the chi-squared test for nominal variables. A p-value less than 0.05 was considered significant. Univariate logistic regression models adjusted for covariates (age; sex; race; comorbidities including human immunodeficiency virus (HIV), hypertension, diabetes, obstructive airway disease, hepatitis B, hepatitis C, congestive heart failure, coronary artery disease and $\mathrm{CKD}$ ) were used to estimate the adjusted odds ratio (AOR) for deaths in patients with AKI versus without AKI. The data obtained was analyzed on IBM's statistical package for the social sciences (SPSS) version 21 .

\section{Results}

Our study analyzed 1545 patient charts of patients admitted to BronxCare hospital from March 2020 through December 2020 with a positive SARS-CoV-2 PCR test. Five of those patients were readmitted after their initial discharge with a positive SARS-CoV-2 PCR test. These charts were counted individually in our statistics. In total, 401 charts had a baseline creatinine from 7 to 365 days prior to admission. For all other patients, we used the MDRD equation to calculate baseline creatinine.

The results of our study analysis show that 608 patients (39.3\%) developed AKI either on admission or during their admission. Of these patients, 137 had underlying chronic renal disease (CKD). A total of 354 patients (58\% of the patients with AKI) expired during their admission, and 190 patients ( $31 \%$ of the patients with AKI) fully recovered from AKI at the time of discharge. The remaining patients were discharged with AKI, primarily stage 1, as determined by the KDIGO criteria. Of our 608 AKI patients, 259 spent time in the ICU. 
Table I Patient Demographics, Comorbidities, and Outcome

\begin{tabular}{|c|c|c|c|c|}
\hline & All $(n=1545)$ & AKI $(n=608)$ & No AKI (n=937) & p Value \\
\hline Age (Avg \pm SD) & $62.63 \pm 15.72$ & $68.48 \pm 13.04$ & $58.82 \pm 16.14$ & $<0.001$ \\
\hline BMI (Avg \pm SD) & $30.11 \pm 7.84$ & $29.95 \pm 7.83$ & $30.22 \pm 7.85$ & 0.304 \\
\hline \multicolumn{5}{|l|}{ Sex, n (\%) } \\
\hline Women & $627(40.6)$ & $229(36.5)$ & $398(63.5)$ & \multirow[t]{2}{*}{0.060} \\
\hline Men & 918 (59.4) & $379(4 I .3)$ & $539(58.7)$ & \\
\hline \multicolumn{5}{|l|}{ Race/ethnicity, n (\%) } \\
\hline Asian & $6(0.4)$ & 0 & $6(0.6)$ & \multirow[t]{5}{*}{$<0.001$} \\
\hline Black & $407(26.3)$ & $194(31.9)$ & $213(22.7)$ & \\
\hline Caucasian & $27(1.7)$ & $10(1.6)$ & $17(1.8)$ & \\
\hline Hispanic & $968(62.7)$ & $353(58.1)$ & $615(65.6)$ & \\
\hline Other or unknown & I37 (8.9) & $5 \mathrm{I}(8.4)$ & $86(9.2)$ & \\
\hline \multicolumn{5}{|l|}{ Comorbidities, n (\%) } \\
\hline HIV & $89(5.8)$ & $40(6.6)$ & $49(5.2)$ & 0.266 \\
\hline HTN & $979(63.4)$ & $457(75.2)$ & $522(55.7)$ & $<0.001$ \\
\hline DM & $732(47.4)$ & $342(56.3)$ & $390(4 I .6)$ & $<0.001$ \\
\hline OAD & $292(18.9)$ & $119(19.6)$ & $173(18.5)$ & 0.586 \\
\hline HBV & $46(3.0)$ & $18(3.0)$ & $28(3.0)$ & 0.975 \\
\hline $\mathrm{HCV}$ & $64(4.1)$ & $32(5.3)$ & $32(3.4)$ & 0.075 \\
\hline $\mathrm{CHF}$ & $167(10.8)$ & $86(14.1)$ & $81(8.6)$ & 0.001 \\
\hline CAD & $179(11.6)$ & $93(15.3)$ & $86(9.2)$ & $<0.001$ \\
\hline CKD & $198(12.8 \%)$ & $137(22.5)$ & $6 \mathrm{I}(6.5)$ & $<0.001$ \\
\hline \multicolumn{5}{|l|}{ Outcome, n (\%) } \\
\hline Discharged & II 03 (7I.4) & $254(4 I .8)$ & $849(90.6)$ & \multirow[t]{2}{*}{$<0.001$} \\
\hline Expired & $442(28.6)$ & $354(58.2)$ & $88(9.4)$ & \\
\hline Day in the hospital (Avg \pm SD) & $9.56 \pm 8.72$ & $11.48 \pm 10.42$ & $8.31 \pm 7.15$ & $<0.001$ \\
\hline
\end{tabular}

Abbreviations: Avg, average; SD, standard deviation; HTN, hypertension; DM, diabetes mellitus; OAD, obstructive airway disease; HBV, hepatitis B virus; HCV, hepatitis $\mathrm{C}$ virus; $\mathrm{CHF}$, congestive heart failure; $\mathrm{CAD}$, coronary artery disease; $\mathrm{CKD}$, chronic kidney disease.

\section{Patient Demographics and Comorbidities}

The majority of the patients in our study were either Hispanic $(62.7 \%)$ or black (26.3\%). About $47.6 \%$ of the black patients developed AKI, while only $36.4 \%$ of the Hispanic patients developed AKI. Out of the 608 patients with AKI $75.2 \%$ of the patients had hypertension and $56.3 \%$ of the patients had diabetes mellitus. Patient demographics, comorbidities, clinical outcomes, and the number of days spent in the hospital are presented in Table 1.

Our results show that there was a statistically significant association between AKI and age, race, hypertension (HTN), diabetes mellitus (DM), coronary artery disease (CAD) congestive heart failure (CHF), CKD, patient outcome, and days spent in the hospital. 


\section{AKI Stages and ICU Admissions}

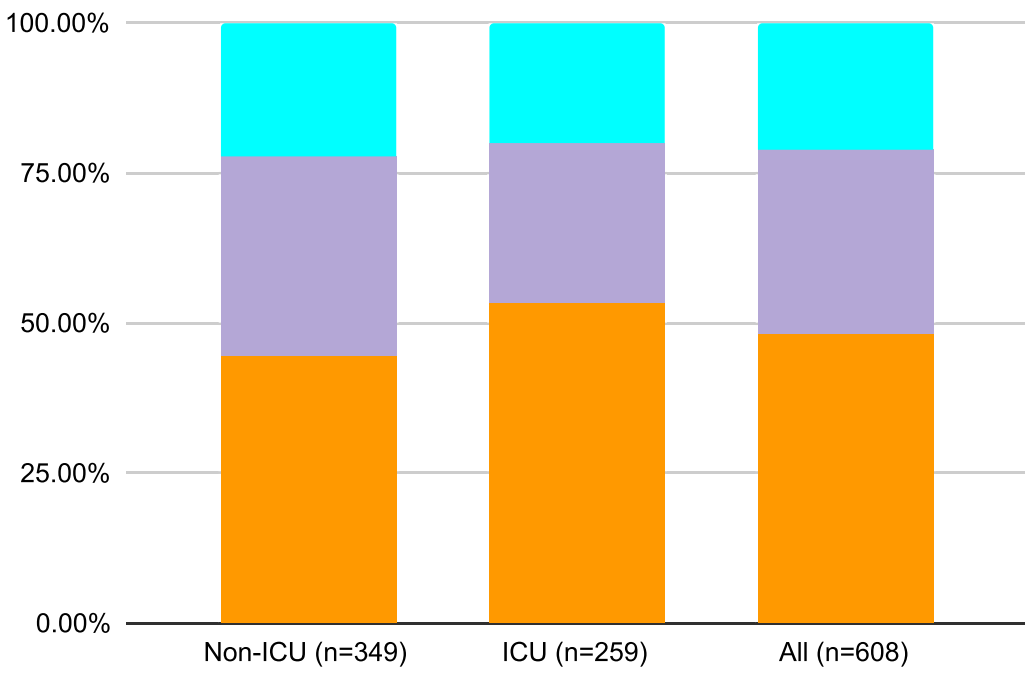

AKI Stage 3

AKI Stage 2

AKI Stage 1

Figure I Acute kidney injury (AKI) stages and intensive care unit (ICU) admission. Abbreviation: $\mathrm{n}$, number of patients.

\section{ICU and AKI Stages}

We further compartmentalized our data looking at how many of our COVID-19 patients with AKI were admitted into the intensive care unit (ICU). Moreover, 42.6\% (259) of patients with AKI were admitted to the ICU. The mean duration of stay in ICU for patients admitted with AKI was $6.17 \pm 5.46$. There was no significant and clinical difference between the stage of AKI and length of ICU stay. Of the 608 patients with AKI, 294 (48.4\%), 185 (30.4\%) and 129 (21.2\%) had stage 1, 2 and 3, respectively. This is presented in Figure 1.

Table 2 Patient Demographics and Characteristics for AKI Patients with and without Prior CKD History

\begin{tabular}{|c|c|c|c|}
\hline & AKI without CKD $(n=47 I)$ & AKI with CKD $(n=137)$ & p Value \\
\hline Age (Avg \pm SD) & $67.52 \pm 13.3$ & $71.76 \pm 11.5$ & 0.001 \\
\hline \multicolumn{4}{|l|}{ Sex (Avg \pm SD) } \\
\hline Male & 285 & 94 & \multirow[t]{2}{*}{0.085} \\
\hline Female & 186 & 43 & \\
\hline BMI (Avg \pm SD) & $29.91 \pm 7.9$ & $30.10 \pm 7.7$ & 0.565 \\
\hline Days in hospital (Avg \pm SD) & $11.78 \pm 10.7$ & $10.48 \pm 11.5$ & 0.12 \\
\hline \multicolumn{4}{|c|}{ HD performed during admission } \\
\hline Yes & 20 & 6 & \multirow[t]{2}{*}{0.946} \\
\hline No & 451 & 131 & \\
\hline \multicolumn{4}{|l|}{ Patient outcome } \\
\hline Discharged & 182 & 72 & \multirow[t]{2}{*}{$<0.001$} \\
\hline Expired & 289 & 65 & \\
\hline
\end{tabular}

Abbreviations: Avg, average; SD, standard deviation; HD, hemodialysis. 
Table 3 Patient Characteristics Associated with Mortality After Adjustment for Age, Sex, Comorbidities, AKI Stage, ICU Admission, and Number of Days Spent in Hospital

\begin{tabular}{|c|c|c|c|}
\hline & Adjusted Odds Ratio & Lower $95 \% \mathrm{Cl}$ & Upper $95 \%$ Cl \\
\hline Age & 0.974 & 0.959 & 0.989 \\
\hline Male & 1.606 & 1.114 & 2.317 \\
\hline HIV & 2.948 & 1.274 & 6.819 \\
\hline HTN & 0.967 & 0.619 & 1.51 \\
\hline DM & 0.718 & 0.499 & 1.035 \\
\hline OAD & 1.613 & 1.004 & 2.593 \\
\hline $\mathrm{HBV}$ & 0.357 & 0.118 & 1.081 \\
\hline $\mathrm{HCV}$ & 0.754 & 0.329 & 1.728 \\
\hline $\mathrm{CHF}$ & 0.897 & 0.52 & 1.548 \\
\hline CAD & 1.125 & 0.666 & 1.9 \\
\hline CKD & 0.501 & 0.324 & 0.774 \\
\hline AKI Stage I & 0.743 & 0.593 & 0.932 \\
\hline ICU Admission & 2.879 & 1.925 & 4.304 \\
\hline Days in Hospital & 1.040 & 1.021 & 1.059 \\
\hline
\end{tabular}

Abbreviations: HTN, hypertension; DM, diabetes mellitus; OAD, obstructive airway Disease; $\mathrm{HBV}$, hepatitis $B$ virus; $\mathrm{HCV}$, hepatitis $\mathrm{C}$ virus; $\mathrm{CHF}$, congestive heart failure; CAD, coronary artery disease; CKD, chronic kidney disease.

\section{AKI vs $A K I$ on CKD}

In this branch of our study, we noted patients with a history of CKD and looked at factors such as age, sex, BMI, days spent in the hospital, hemodialysis, and patient outcome.

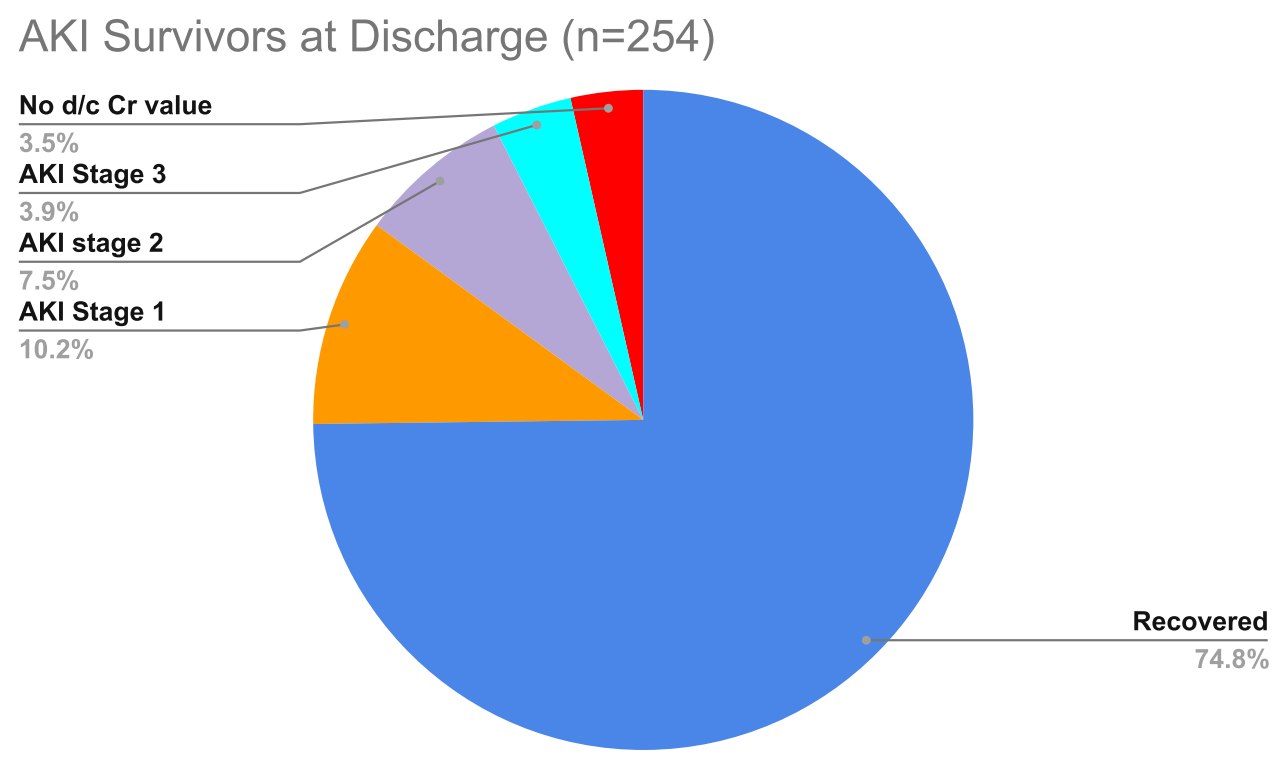

Figure 2 AKI survivors at discharge $(n=254)$.

Abbreviations: $n$, number of patients; D/c, discharge; $\mathrm{Cr}$, creatinine. 
We found that out of the 608 patients with AKI, 471 had no prior history of CKD. Twenty-six patients required hemodialysis (HD) during the hospitalization, four of them expired.

The mortality rate was $58.2 \%$ (354). Of those with acute on chronic kidney injury, $47.4 \%$ expired, while $61.36 \%$ of AKI patients without prior CKD expired. Results comparing AKI and AKI on CKD can be seen in Table 2.

\section{$\mathrm{AKI}$ and Mortality Rates}

Approximately $58.2 \%$ of AKI patients expired. We adjusted specific demographics and comorbidities associated with mortality in Table 3. Male gender, history of HIV, history of OAD and ICU admissions were significantly associated with mortality.

The adjusted odds ratio for death was 13.0 (95\% CI, 7.2 to 18) for all patients with AKI versus no AKI. Of the 254 patients who survived, $74.8 \%$ of the patients had a complete recovery. Seventy-two of the discharged patients had CKD at baseline and the majority (63.8\%) made a complete recovery. We stratified the remaining patients by AKI stage at discharge based on the ratio of baseline creatinine to discharge creatinine. Nine of our patients did not have a discharge creatinine value within 48 hours of discharge. Results are in Figure 2.

\section{Discussion}

In approximately 1500 patients admitted to BronxCare Hospital with COVID-19, 39\% developed AKI, and 58.2\% of those patients expired during their time in the hospital. Of the patients who survived, the majority made a full recovery, determined by creatinine levels returning to baseline. Moreover, $42.6 \%$ of AKI patients were admitted to the ICU. The rate of AKI in our patient population was significantly higher than that reported in studies across the globe. ${ }^{4}$ However, when looking at the results of other studies performed locally, we find that our numbers are comparable. This could be due to the high prevalence of AKI risk factors in our patient population, such as age, race, hypertension (HTN), diabetes mellitus (DM), coronary artery disease (CAD), congestive heart failure (CHF) and CKD which all showed a statistically significant association in our results.

The incidence of AKI in COVID-19 is higher than in the most recent respiratory pandemic: influenza A H1N1 in 2009. According to a meta-analysis performed on 20 studies and looking at over 3000 patients with H1N1 who developed AKI, 33\% of the patients admitted with H1N1 developed AKI. The study demonstrated results comparable with ours, showing an association with specific comorbidities such as obesity and diabetes. ${ }^{10}$ Furthermore, the histology assessment of the kidneys of H1N1-infected patients also demonstrated acute tubular necrosis (ATN), DIC, and myoglobin pigments, ${ }^{11}$ showing that tubular necrosis may be the common mechanism of renal injury of patients infected with these respiratory illnesses.

The pathogenesis of AKI in COVID-19 is multifactorial. The most common cause of acute kidney injury in COVID19 patients was acute tubular injury that resulted from. ${ }^{12}$ Direct viral infection and replication in podocytes and tubules of kidney cells was shown to cause tubular injury. Prominent acute proximal tubular necrosis was observed by light microscopy, with loss of brush border, non-isometric vacuolar degeneration and even frank necrosis. ${ }^{13}$ Subsequently, endothelial injury, microvascular thrombi, local inflammation and immune cell infiltration have been repeatedly observed in patients with COVID-19 AKI. ${ }^{14}$ The overloaded immune response and cytokine storm cause acute tubular necrosis and endothelial injury that may result in a thrombus. ${ }^{13}$ This in conjunction with observed erythrocyte aggregates obstructing the lumen of capillaries indicates that abnormal coagulation may also participate in the pathogenesis of AKI in COVID-19. ${ }^{13}$ Multiple indirect causes or insulting events also play a role in contributing to AKI in COVID-19 patients, such as medications, hemodynamic instability, hypoxia, acute respiratory distress syndrome, rhabdomyolysis, excessive diuresis, and secondary infections. ${ }^{15}$

Our study has a few limitations, the first being that more than half of the charts we analyzed did not have a baseline creatinine value. We therefore resorted to the MDRD equation to calculate one and determine whether or not the patient had AKI. Additionally, certain discharged patients were excluded from the final numbers tracking recovery status due to a lack of creatinine values for comparison. Lastly, if a patient did not have AKI upon admission, in that case, it is impossible to ascertain whether the renal injury was caused by COVID-19, the multitude of medications they received during admission or/and any acute events they may have suffered from while in the hospital. However, it can be assumed that many patients worldwide have received similar antiviral and antibiotic treatments. 


\section{Conclusion}

The results of our study incite us to believe that there may be a correlation between COVID-19 and the development of AKI. Preventative measures should be considered for patients admitted with COVID-19. As the long-term effects on the kidneys are unknown, resource planning and strategies to improve the long-term understanding and kidney care are necessary. Nephrologists should be consulted early and nephrotoxic agents should be avoided. Finally, discharge planning with a follow-up nephrology appointment should be considered to mitigate the onset of AKI.

\section{Ethics Statement}

The BronxCare Health System Institutional Review Board (IRB) reviewed and approved our study. A waiver of the HIPAA authorization was granted by the IRB as this is a minimal risk retrospective chart study. Therefore, informed consent was not required. The guidelines noted in the Declaration of Helsinki were followed.

\section{Disclosure}

The authors report no conflicts of interest in this work.

\section{References}

1. CDC COVID Data Tracker. Centers for Disease Control and Prevention. Available from: https://covid.cdc.gov/covid-data-tracker/. Accessed February 22, 2022.

2. Vacchi C, Meschiari M, Milic J, et al. Covid-19-associated vasculitis and thrombotic complications: from pathological findings to multidisciplinary discussion. Rheumatology. 2020;59(12):e147-e150. doi:10.1093/rheumatology/keaa581

3. McGonagle D, Bridgewood C, Ramanan AV, Meaney JF, Watad A. Covid-19 vasculitis and novel vasculitis mimics. Lancet Rheumatol. 2021;3(3). doi:10.1016/s2665-9913(20)30420-3

4. Chen Y-T, Shao S-C, Hsu C-K, Wu I-W, Hung M-J, Chen Y-C. Incidence of acute kidney injury in COVID-19 infection: a systematic review and meta-analysis. Critical Care. 2020;24(1). doi:10.1186/s13054-020-03009-y

5. Chan L, Chaudhary K, Saha A, et al. AKI in hospitalized patients with Covid-19. J Am Soc Nephrol. 2020;32(1):151-160. doi:10.1681/ asn. 2020050615

6. Hughes PJ, Desai T, Lerma EV. Classification systems for acute kidney injury: background, rifle classification, acute kidney injury network. Classification Systems for Acute Kidney Injury; 2021. Available from: https://emedicine.medscape.com/article/1925597-overview. Accessed February 22, 2022.

7. American Kidney Fund. Acute kidney injury \& failure (AKI) symptoms, causes, \& treatments; 2021. Available from: https://www.kidneyfund.org/ kidney-disease/kidney-problems/acute-kidney-injury.html. Accessed February 22, 2022.

8. Kidney International Supplements. KDIGO clinical practice guideline for acute kidney injury. Off J Int Soc Nephrol. 2012;2(1):1. doi:10.1038/ kisup.2012.1

9. Ali AY, Elkhashab SO. Cystatin: assessment of renal function in chronic kidney disease and postrenal transplant patients. $J$ Egypt Soc Nephrol Transplant. 2019;19(3):75. doi:10.4103/jesnt.jesnt_22_18

10. Strohbehn IA, Zhao S, Seethapathy H, et al. Acute kidney injury incidence, recovery, and long-term kidney outcomes among hospitalized patients with COVID-19 and influenza. Kidney Int Rep. 2021;6(10):2565-2574. doi:10.1016/j.ekir.2021.07.008

11. Martin-Loeches I, Papiol E, Rodríguez A, et al. Acute kidney injury in critical ill patients affected by influenza A (H1N1) virus infection. Crit Care. 2011;15(1). doi:10.1186/cc10046

12. Nugent J, Aklilu A, Yamamoto Y, et al. Assessment of acute kidney injury and longitudinal kidney function after hospital discharge among patients with and without COVID-19. JAMA Network Open. 2021;4(3):e211095. doi:10.1001/jamanetworkopen.2021.1095

13. Qian J-Y, Wang B, Lv LL, Liu B-C. Pathogenesis of acute kidney injury in coronavirus disease 2019. Front Physiol. 2021;12. doi:10.3389/ fphys.2021.586589

14. Legrand M, Bell S, Forni L, et al. Pathophysiology of COVID-19-associated acute kidney injury. Nat Rev Nephrol. 2021;17(11):751-764. doi: 10.1038/s41581-021-00452-0

15. Santoriello D, Khairallah P, Bomback AS, et al. Postmortem kidney pathology findings in patients with COVID-19. J Am Soc Nephrol. 2020;31 (9):2158-2167. doi:10.1681/asn.2020050744

\section{Publish your work in this journal}

The International Journal of Nephrology and Renovascular Disease is an international, peer-reviewed open-access journal focusing on the pathophysiology of the kidney and vascular supply. Epidemiology, screening, diagnosis, and treatment interventions are covered as well as basic science, biochemical and immunological studies. The manuscript management system is completely online and includes a very quick and fair peer-review system, which is all easy to use. Visit http://www.dovepress.com/testimonials.php to read real quotes from published authors.

Submit your manuscript here: https://www.dovepress.com/international-journal-of-nephrology-and-renovascular-disease-journal 